GENERAL COST STRUCTURE ANALYSIS

Theory and Application to the Banking Industry 


\title{
GENERAL COST STRUCTURE ANALYSIS
}

Theory and Application to the Banking Industry

\author{
by \\ Ziad Sarkis \\ University of Oxford
}

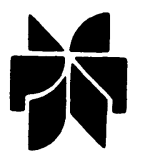

Springer Science+Business Media, LLC 


\section{Library of Congress Cataloging-in-Publication Data}

Sarkis, Ziad.

General cost structure analysis : theory and application to the banking industry / by Ziad Sarkis.

p. $\mathrm{cm}$.

Includes bibliographical references and index.

ISBN 978-1-4613-7078-9 ISBN 978-1-4615-4599-6 (eBook)

DOI 10.1007/978-1-4615-4599-6

1. Banks and banking -- Costs -- Econometric models. 2. Cost accounting. I. Title.

HG1588.S25 1999

$332.1^{\prime} 068^{\prime} 1--\mathrm{dc} 21$

Copyright $\odot 1999$ by Springer Science+Business Media New York

Originally published by Kluwer Academic Publishers, New York in 1999

Softcover reprint of the hardcover 1st edition 1999

All rights reserved. No part of this publication may be reproduced, stored in a retrieval system or transmitted in any form or by any means, mechanical, photo-copying, recording, or otherwise, without the prior written permission of the publisher, Springer Science+Business Media, LLC .

Printed on acid-free paper. 
To my family for so much

unwavering encouragement and unconditional support 


\section{Contents}

1 Introduction 1

2 Theoretical Framework 5

2.1 Generalised Firm Program . . . . . . . . . . . . . 7

2.2 Previous Approaches . . . . . . . . . . . . . . 8

2.3 Flexible Cost Model (FCM) . . . . . . . . . . . . . 11

2.3.1 Primal Characterisation . . . . . . . . . . 11

2.3.2 Dual Characterisation: Globally Regular Functional Forms . . . . . . . . . . . . 13

2.3.3 Dual Characterisation: General Case . . . . . 15

2.4 Comparative Statics . . . . . . . . . . . . 20

3 Empirical Modelling 23

3.1 Empirical Context and Issues . . . . . . . . . . . . 25

3.2 Output and Input Measurement . . . . . . . . . 27

3.3 Dataset . . . . . . . . . . . . . . . 29

3.4 Empirical Specification . . . . . . . . . . . . . 37

3.4.1 Parametric Specification . . . . . . . . . . 37

3.4.2 Stochastic Specification and Estimation Methodology . . . . . . . . . . . . . . 37

3.4.3 Dual Characterisation . . . . . . . . . . . . 40 
viii Contents

4 Empirical Results $\mathbf{4 5}$

4.1 Econometric Results . . . . . . . . . . . . . . . 47

4.1.1 Single Equation Estimation: Core Results . . . 47

4.1.2 Single Equation Estimation: Restrictions and Extensions . . . . . . . . . . 50 50

4.1 .3 System Estimation . . . . . . . . . . . . . . 52

4.1.4 Evidence of Output Measurement Error . . . . 54

4.2 Scale Effects . . . . . . . . . . . . . . . . 54

4.2.1 Primal Measurement . . . . . . . . . . . . 56

4.2 .2 Dual Measurement . . . . . . . . . . . . . . 58

4.3 Capacity Utilisation Effects . . . . . . . . . . . 63

4.3.1 Allocative Efficiency Indices . . . . . . . . . . 63

4.3.2 Capacity Utilisation Rates . . . . . . . . . . . . 68

4.3.3 Economic Consequences . . . . . . . . . . . 78

4.4 Impact of Labour Market Rigidities . . . . . . . . . 83

4.5 Sources of International Cost Position Differences . . . 84

$\begin{array}{lll}5 & \text { Conclusion } & 89\end{array}$

6 Bibliography $\quad 93$

7 Appendix: Dataset Statistics 101

$\begin{array}{ll}\text { Index } & 117\end{array}$ 


\section{Acknowledgments}

I wish to express my particular gratitude to: Kenneth Arrow, for his ever kind and patient guidance; Richard Smethurst and John Vickers, who generously opened Oxford's doors to me; Colin Mayer, for his essential counsel and insightful observations; Steve Bond and Bronwyn Hall, to whom I owe a considerable intellectual debt; and Allard Winterink, senior editor at Kluwer Academic Publishers, for his remarkable efficiency.

Naturally, the responsibility for any errors or inaccuracies rests solely with me. 


\section{LIST OF FIGURES}

3.1. Evolution of price of labour .................... 33

3.2. Evolution of price of technology .................. 34

3.3. Evolution of mean labour/technology ratio ........... 35

3.4. Cost/income ratio vs. number of employees........... 36

4.1. Evolution of mean production function residual..........55

4.2. Scale effects metric vs. number of employees...........57

4.3. Cost/income ratio vs. logarithm of output value: first derivative. ........ 59

4.4. Cost/income ratio vs. logarithm of output value: second derivative .......60

4.5. Cost/income ratio vs. number of employees: ceteris paribus relation ....... 62

4.6. Evolution of mean value of first-order condition residual...................64

4.7. Distribution of first-order condition residual ..........667

4.8. Production function residual vs. first-order condition residual . .................69

4.9. Production function residual vs. first-order condition residual: first sub-sample .......70

4.10. Production function residual vs. first-order condition residual: second sub-sample . . . 71

4.11. Output approximation error: first-order Taylor series . . . 73 4.12. Output approximation error: second-order Taylor series.. 74 4.13. Output approximation error: third-order Taylor series . . 75 4.14. Labour capacity utilisation rate vs. first-order condition residual $\ldots \ldots \ldots \ldots \ldots \ldots \ldots \ldots$

4.15. Cost/income ratio vs. first-order condition residual: first derivative $\ldots \ldots \ldots 80$

4.16. Cost surcharge vs. first-order condition residual........ 81 


\section{LIST OF TABLES}

3.1. Mean value of cost/income ratio ..................37

4.1. Production function estimation: results .............. 48

4.2. Production function estimation: notes .............. 49

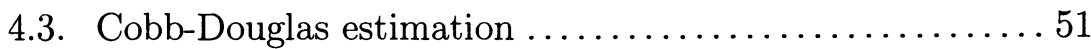

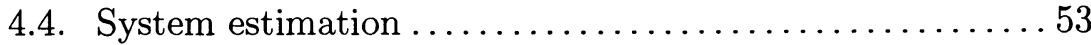

4.5. Mean value of

first-order condition residual: detail $\ldots \ldots \ldots \ldots \ldots \ldots 66$

4.6. Mean output approximation error .................. 76

4.7. Mean value of labour capacity utilisation rate ......... 77

4.8. Mean value of technology capacity utilisation rate ...... 78

4.9. Mean value of cost surcharge ................... 82

4.10. Impact of labour market rigidities . . . . . . . . . . . 83

4.11. Sources of cost differences: first sub-sample .......... 85

4.12. Sources of cost differences: second sub-sample ........ 86

7.1. Number of observations by sub-sample ............ 103

7.2. Observations meeting production function regularity conditions.............. 104

7.3. Observations meeting all regularity conditions ......... 105

7.4. Selected firm-level statistics: France............... 106

7.5. Selected firm-level statistics: Germany...............108

7.6. Selected firm-level statistics: Japan.................. 109

7.7. Selected firm-level statistics: UK . . . . . . . . . . . . 110

7.8. Selected firm-level statistics: US . . . . . . . . . . . . . 112 